\section{Complement C5-blocking Agent in Refractory Dermatomyositis}

To the Editor:

Dermatomyositis (DM) is a rare autoimmune disease that belongs to the group of idiopathic inflammatory myopathies (IIM). The usual treatment of DM was based on steroids, methotrexate, and azathioprine. Intravenous immunoglobulin (IVIG), rituximab (RTX), or other biological treatments recently emerged as steroid-sparing agents ${ }^{1}$. About $10 \%$ to $30 \%$ of patients will develop a refractory or chronic persistent disease ${ }^{2}$, suggesting that alternative treatments should be tested. Here, we report a case of acute refractory DM with concomitant thrombotic microangiopathy (TMA) that improved with the C5-blocking agent eculizumab in the setting of other medications.
According to the French law regarding observational retrospective studies (Jardé Law, 2016), written informed consent to publish the material was obtained from the patient, but approval by a specific ethics board was waived.

A 19-year-old female patient, without medical history or medications, developed moderate arm and limb muscle weakness, Raynaud phenomenon, Gottron papules, mechanic's hand, and edema of limb, arm, and periorbital regions. One month later, she was admitted to the hospital for severe acrocyanosis (Figure 1A-B), and examination showed elevated muscle enzymes [creatine kinase (CK) $11.243 \mathrm{IU} / \mathrm{l}$, myoglobin $10.152 \mathrm{ng} / \mathrm{ml}$, troponin $7.404 \mathrm{ng} / \mathrm{l}]$. Cardiac magnetic resonance imaging (MRI) showed left ventricle hypertrophy and pericardial effusion suggestive of myocarditis, but breath-related artifacts precluded definitive diagnosis. Capillaroscopy
A

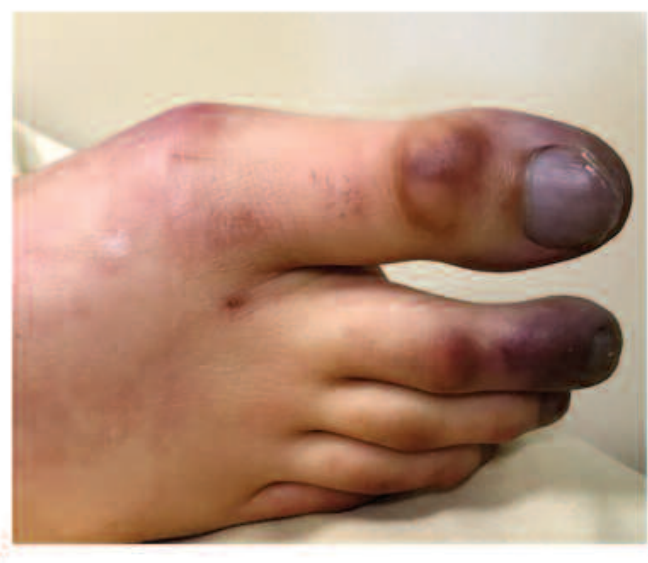

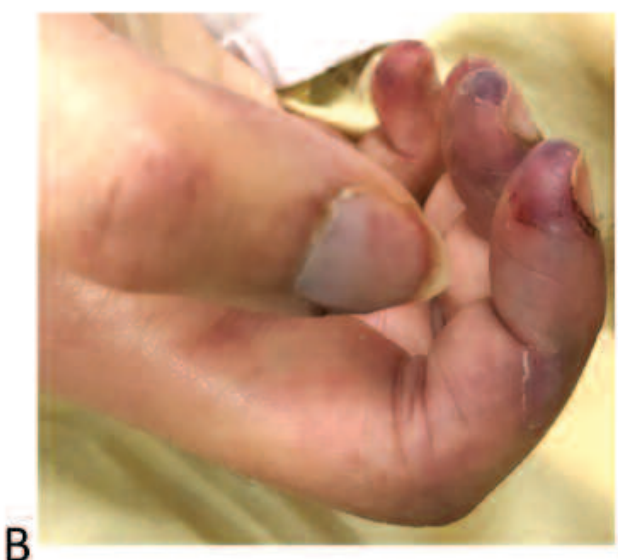

$\downarrow \quad \downarrow \quad \downarrow \quad$ Rituximab

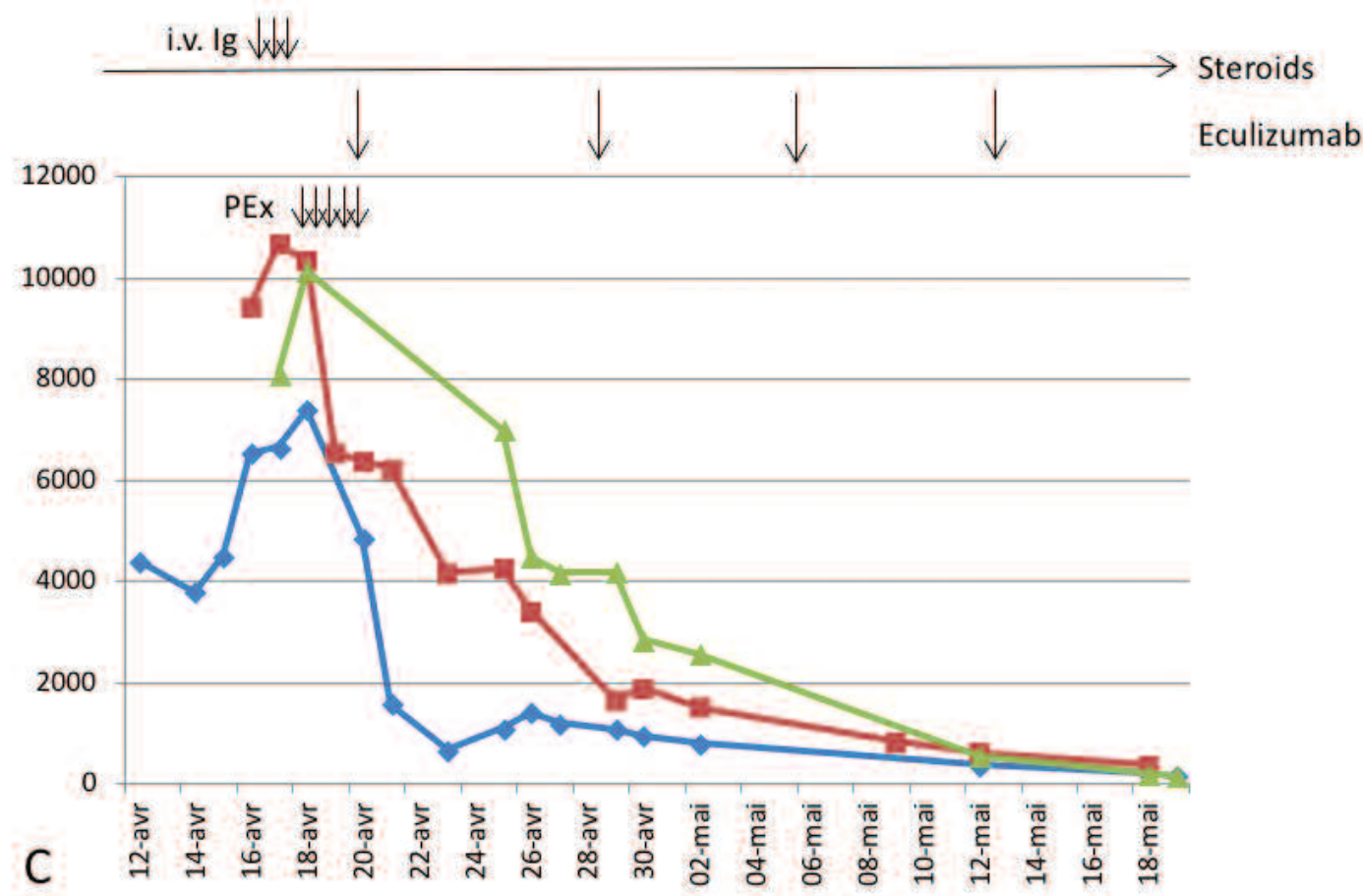

Figure 1. A and B. Severe acrocyanosis of the fingers and toes, with Gottron papules and scleroderma. C. Kinetics of CPK (red; IU/l), myoglobin (green; ng/ml), and troponin (blue, ng/l). IVIg: intravenous immunoglobulins; CPK: creatine phosphokinase; PEx: plasma exchange. 
demonstrated abnormal tortuous nailfold capillaries. Immunological tests showed only low serum complement $\mathrm{C} 3 / \mathrm{C} 4$ components and increased soluble C5b-9 (Supplementary Table 1, available from the authors on request), but no antinuclear antibodies and myositis-specific autoantibodies. Neuromuscular biopsy showed perivascular and perimysial infiltration of nonnecrotic muscle fibers with CD4+ T cell and CD68+ macrophages, MHC-1 antigen expression close to the inflammatory infiltrates, and focal perifascicular atrophy suggestive of seronegative $\mathrm{DM}^{3}$. At that time, she had arthralgia but no arthritis, dysphagia, or interstitial lung disease. Skin biopsy and muscular MRI were not performed. Diagnosis of DM was retained according to the Bohan and Peter criteria (skin lesions and 3 out of 4 major criteria $)^{4}$. Methylprednisolone $(2 \mathrm{mg} / \mathrm{kg} /$ day) was started and IVIG was added at Day 3 because of persistent pain and edema of the legs, arms, and face, and elevated $\mathrm{CK}$

At Day 4, the patient was referred to our intensive care unit (ICU) for acute kidney injury with glomerular presentation (serum creatinine 278 $\mu \mathrm{mol} / 1$, protein-to-creatinine ratio $2.9 \mathrm{~g} / \mathrm{g}$, hematuria, hypertension) and features of TMA (hemolytic anemia, thrombocytopenia, decreased haptoglobin, lactate dehydrogenase increase, and circulating schizocytes). Search for anti-ADAMTS13, anticardiolipins, and anti- $\beta_{2}$-GPI antibodies and genetic analysis of complement genes was negative. ADAMTS13 activity was within normal ranges (58\%). IVIG were withdrawn. Despite daily plasma exchanges from Day 4 to Day 8, the patient developed extensive severe acrocyanosis, anuria, and status epilepticus, prompting us to use salvage therapy by eculizumab (Day $8 ; 900 \mathrm{mg}$ weekly for 1 month, then followed by $1200 \mathrm{mg}$ every 14 days) after pneumococcal and meningococcal vaccination ${ }^{5}$. Antibioprophylaxis by ceftriaxone was also started and steroids were maintained. Plasma exchanges were stopped.

As shown in Figure 1C, whereas CK and myoglobin levels were slightly or not modified by steroids, IVIG, and plasma exchanges, the first infusion of eculizumab was followed by a dramatic control of the muscle edema and myalgia. It was also followed by a complete reversal of the distal cyanosis and rapid decline in CK and myoglobin levels. Despite the lack of myositis-specific autoantibodies, RTX was given from days 18 to 40 (375 $\mathrm{mg} / \mathrm{m}^{2}$ weekly).

Mechanical ventilation and dialysis were withdrawn at days 21 and 28, respectively. Eculizumab was stopped at Month 4 and RTX $500 \mathrm{mg}$ was infused every 6 months. Six months after the admission to the ICU, serum creatinine was $80 \mu \mathrm{mol} / 1$, and proteinuria and hematuria were negative. Hypertension was controlled using angiotensin-converting enzyme inhibitor. CK and myoglobin were in normal ranges and the patient did not report muscle weakness. Skin examination was normal. Search for neoplasia was negative.

Data from pathological studies suggest that DM is characterized by an antigen activation of the $\mathrm{C} 5 \mathrm{~b} 9$ macrophage-activating complex and deposition on the surface of the endothelial cells, with subsequent capillary ischemia and rarefaction, and CD4+ T cells infiltration ${ }^{6,7}$. Primary and secondary hemolytic and uremic syndromes (HUS), forms of TMA, are also characterized by an uncontrolled activation of the alternative complement pathway. Interestingly, a case of HUS was previously described in a patient with an IIM ${ }^{8}$, reinforcing the potential link between IIM and HUS forms of TMA. In contrast, direct involvement of skeletal muscle is rare in patients with $\mathrm{TMA}^{9}$. In this case, we successfully used eculizumab as a salvage therapy for a refractory HUS/TMA ${ }^{10}$, with concomitant severe DM. Other immunosuppressive agents helped to control the DM, but the almost complete normalization of the CK level after the first 2 infusions of eculizumab (before the use of RTX), contrasting with the lack of rapid response following steroids and plasma exchange, strongly suggested that eculizumab was an effective treatment to add-on in severe DM. This finding was similar to that of a previous study that included 10 patients with refractory $\mathrm{DM}^{11}$.

Our case suggests that $\mathrm{C} 5$ blocking should be tested in life-threatening or refractory DM.

STANISLAS FAGUER — , MD, PhD, Département de Néphrologie et Transplantation d'organes, and Centre de référence des maladies rénales rares, Centre Hospitalier de Toulouse; JULIE BELLIERE, MD, PhD, Département de Néphrologie et Transplantation d'organes, and Centre de référence des maladies rénales rares, Centre Hospitalier de Toulouse; DAVID RIBES, MD, Département de Néphrologie et Transplantation d'organes, and Centre de référence des maladies rénales rares, Centre Hospitalier de Toulouse. Address correspondence to Professor S. Faguer, Département de Néphrologie et Transplantation d'organes, INSERM U1048 (équipe 12, I2MC), Hôpital Rangueil 1, Avenue Jean Poulhes, 31000 Toulouse, France. E-mail: faguer.s@chu-toulouse.fr

\section{REFERENCES}

1. Leclair V, Lundberg IE. Recent clinical trials in idiopathic inflammatory myopathies. Curr Opin Rheumatol 2017;29:652-9.

2. Oddis CV, Reed AM, Aggarwal R, Rider LG, Ascherman DP, Levesque MC, et al. Rituximab in the treatment of refractory adult and juvenile dermatomyositis and adult polymyositis: a randomized, placebo-phase trial. Arthritis Rheum 2013;65:314-24.

3. Mandel DE, Malemud CJ, Askari AD. Idiopathic inflammatory myopathies: a review of the classification and impact of pathogenesis. Int J Mol Sci 2017;18:1084.

4. Bohan A, Peter JB. Polymyositis and dermatomyositis. N Engl J Med 1975;292:344-7.

5. Winthrop KL, Mariette X, Silva JT, Benamu E, Calabrese LH, Dumusc A, et al. ESCMID Study Group for Infections in Compromised Hosts (ESGICH) Consensus Document on the safety of targeted and biological therapies: an infectious diseases perspective (Soluble immune effector molecules [II]: agents targeting interleukins, immunoglobulins and complement factors). Clin Microbiol Infect 2018;24 Suppl 2:S21-40.

6. Lahoria R, Selcen D, Engel AG. Microvascular alterations and the role of complement in dermatomyositis. Brain 2016;139:1891-903.

7. Pytel P. C4d staining as immunohistochemical marker in inflammatory myopathies. Appl Immunohistochem Mol Morphol 2014;22:696-704

8. Couvrat-Desvergnes G, Masseau A, Benveniste O, Bruel A, Hervier $\mathrm{B}$, Mussini JM, et al. The spectrum of renal involvement in patients with inflammatory myopathies. Medicine 2014;93:33-41.

9. Hofer J, Rosales A, Fischer C, Giner T. Extra-renal manifestations of complement-mediated thrombotic microangiopathies. Front Pediatr 2014;2:97.

10. Licht C, Greenbaum LA, Muus P, Babu S, Bedrosian CL, Cohen DJ, et al. Efficacy and safety of eculizumab in atypical hemolytic uremic syndrome from 2-year extensions of phase 2 studies. Kidney Int 2015;87:1061-73

11. Takada K, Bookbinder S, Furie R, Oddis C, Mojcik C, Bombara M, et al. A pilot study of eculizumab in patients with dermatomyositis. Arthritis Rheum 2002;46 Suppl 9:S489.

J Rheumatol First Release September 152018 ; doi:10.3899/jrheum. 180060 